\title{
Cross-Cultural Study of Emotional Expression: The Problem of Alexithymia*
}

\author{
Makhinur Mamatova \\ American University of Central Asia, \\ Bishkek, Kyrgyzstan
}

\author{
Diane E. Wille \\ Indiana University Southeast, \\ New Albany, USA
}

\begin{abstract}
The present study is a cross-cultural investigation of alexithymia both state and trait stress. Participants from the USA of America and Kyrgyzstan, a Former Soviet Republic, which is primarily Islamic with a nomadic history, completed the Toronto Alexithymia Scale (Bagby, Parker, \& Taylor, 1994) and the State-Trait Anxiety Inventory for Adults (Spielberger, 1983). As expected, individuals with higher levels of alexithymia reported greater state and trait anxiety. Compared with the USA sample, Kyrgyz participants reported higher levels of alexithymia, however, no country differences were found for level of stress. Further analysis revealed that both country and trait anxiety were robust predictors of alexithymia. The etiology of culturally normative alexithymia is discussed.
\end{abstract}

Keywords: cross-cultural research, alexithymia, state and trait stress

\section{Introduction}

This study is the first step in the investigation of cross-cultural aspects of self-expression, including its emotional affective components. Self-expression as the subject of psychological investigation is one of the developing topics in the current field of psychology. In the most general formulation of emotional communication, there are three ways to express oneself: socially, physically and psychologically. Alexithymia, as a psychological dimension of emotion, is selected as the operational unit in the current study to research individual differences in emotional expressions.

Alexithymia is described as the infringement of the ability to recognize and verbally describe one's own emotions (Nemiah, Freyberger, \& Sifneos, 1976). The alexithymia construct is formulated by Nemiah et al. (1976) on the basis of clinical observations and is comprised of the following features: (1) difficulty identifying feelings; (2) difficulty describing feelings; (3) limited imagination capacity; and (4) an externally oriented way of thinking.

The study of the problems of alexithymia is an actively developing field of clinical psychology. Researchers in this area focus their attention on the role of alexithymia in the etiology of psychosomatic disorders, anxiety disorders, somatoform disorders, and depression. Empirical evidence shows that alexithymia is associated with difficulties in discriminating among different emotional states (Bagby, Parker, \& Taylor, 1994), and with a limited ability to think about and use emotions to cope with stressful situations (Parker,

\footnotetext{
*Acknowledgement: The authors wish to thank the participants of this study and Indiana University Southeast for a Grant-in-Aid.

Makhinur Mamatova, Ph.D., acting associate professor, Department of Psychology, American University of Central Asia.

Diane E. Wille, Ph.D., Professor, School of Social Sciences, Indiana University Southeast.
} 
Taylor, \& Bagby, 1998). The limited ability to represent and regulate emotions cognitively is thought to render alexithymic individuals more susceptible to a variety of medical and psychiatric illnesses (Taylor, Bagby, \& Parker, 1997).

Alexithymia is not only a clinical phenomenon. Inability to recognize one's own emotions does not necessarily mean that the person is unable to experience emotions. Alexithymia is considered to be a personality dimension with still unknown origins. Researchers show that alexithymia is normally distributed in the general population in both genders (Salminen, Saarijärvi, Äärelä, Toikka, \& Kauhanen, 1999). However, there are still few comparative population studies on the prevalence of alexithymic characteristics. Alexithymic traits within the context of personality profile are not the symptoms of the clinical problem. Though these traits might predispose a person to the onset of specific interpersonal problems, such as distant and non-assertive social functioning (Vanheule, Desmet, Megank, \& Bogarets, 2007).

Research on the etiology of alexithymia suggests that it is a culturally related phenomenon. The relationship between alexithymia and culture was examined in several cross-cultural studies in college students from three cultural groups: European-Americans, Asian-Americans and Malaysians (Le, Berenbaum, \& Raqhavan, 2002). European-American students showed lower alexithymia index than both Asian groups. Further exploration of the cultural roots of alexithymia is necessary to determine which aspects of culture play a role in its development.

The current study will use measures of alexithymia to investigate emotional aspects of self-expression in two cultures: the USA and Kyrgyzstan. Kyrgyzstan is a Former Soviet Republic located in Central Asia. The predominant religion is Islam. The historical milieu is characterized by nomadism. Kyrgyz and Russian are two major languages. Kyrgyzstan is a multiethnic state, which is home to 90 different ethnic groups. The authors hypothesize that the cultural aspects of the Kyrgyz culture (Former Soviet Republic, Islamic and nomadism) will create greater emotional constraints and the Kyrgyz subjects will show a higher rate of alexithymia than the participants from the United States.

The association between alexithymia and anxiety has been investigated in several studies which found significant correlations between them (Karukivi et al., 2010). The relationship between culture and stress (both state and trait) will also be investigated in this study. A positive relationship is expected between alexithymia and stress. However, cultural differences in stress level are not expected. Research has not found a consistent link between stress and culture (Asmal \& Stein, 2009).

\section{Methods}

\section{Participants}

Thirty-nine individuals from Kyrgyzstan, 21 males and 18 females, and 25 from the United States, 12 male and 13 female, participated in this study. The Kyrgyz sample was recruited from a mid-sized university in the capital city of Bishkek, Kyrgyzstan. The USA sample was recruited from a mid-sized university in the Louisville, Kentucky metro area. The predominant ethnic background of the individuals from Kyrgyzstan was Kyrgyz and European American for the sample from the USA (see Table 1). Each sample reflects the major religion of the country, the Kyrgyzstan participants were predominantly Muslim and the USA participants were Christian, either Catholic or Protestant. The Kyrgyzstan participants were primarily sophomore level college students, whereas the participants from the USA were freshman, however, mean ages of the two groups were similar since children in Kyrgyzstan graduate from high school a year earlier than in the USA. 
Table 1

Study Participants

\begin{tabular}{|c|c|c|c|}
\hline & & Kyrgyzstan & USA \\
\hline \multirow{2}{*}{ Gender } & Male & 21 & 12 \\
\hline & Female & 18 & 13 \\
\hline \multirow{5}{*}{$\begin{array}{l}\text { Ethnic } \\
\text { background }\end{array}$} & Kyrgyz & 26 & \\
\hline & Russian & 5 & \\
\hline & Korean & 3 & \\
\hline & Uzbek & 4 & \\
\hline & European-American & & 25 \\
\hline \multirow{5}{*}{ Religion } & Muslim & 25 & \\
\hline & Orthodox & 7 & \\
\hline & Catholic & & 10 \\
\hline & Protestant & & 11 \\
\hline & Freshman & 3 & 17 \\
\hline \multirow{3}{*}{ Year in school } & Sophomore & 31 & 6 \\
\hline & Junior & 5 & 2 \\
\hline & Age (mean) & 19.13 & 19.28 \\
\hline
\end{tabular}

\section{Procedures}

The participants completed the TAS (Toronto alexithymia scale) (Bagby et al., 1994) and the STAI (state-trait anxiety inventory for adults) (Spielberger, 1983). Each group of students were administered the assessments in their native language. For the participants in Kyrgyzstan it was Russian; for the participants from the USA, English.

The TAS-20 (Toronto Alexithymia Scale) (Bagby et al., 1994) consists of 20 items which are rated on a five-point Likert scale ranging from 1 (“Strongly agree”) to 5 (“Strongly disagree”). The participants' responses were reverse scored so that a higher score indicated less understanding of one's feelings and greater alexithymia. Items were then summed and averaged to create an alexithymia score for each participant. The TAS-20 has been found to be a reliable and valid instrument and has been used widely in diverse cultures (Bagby et al., 1994; Güleç, Köse, Güleç, Çitak, Evren, Borckardt, \& Sayar, 2009; Le, Berenbaum, \& Raghavan, 2002; Taylor et al., 2003; Taylor, Bagby, Ryan, \& Parker, 1988)

The STAI (state trait anxiety inventory) (Spielberger, 1983) assesses anxiety that is either created by the environment (state) or is an internal personality characteristic (trait). The STAI contains 40 items which are rated on a four-point Likert scale ranging from 1 (“Not at all”), 2 (“Somewhat”), 3 ("Moderately so”), and 4 ("Very much so"). Twenty items assess state and twenty items assess trait anxiety. The standardized procedures are used to create a state and trait anxiety score for each participant. Higher scores indicate greater state and trait anxiety. The STAI has been used extensively in cross-cultural research and has been found to be both reliable and valid in these contexts (Hanin \& Spielberger, 1983).

\section{Results}

Pearson correlations were employed to determine the relation between TAS and both state and STAI scores for the participant sample as a whole. Greater alexithymia was found to predict greater state, $r_{(63)}=0.44$, $p<0.01$, and trait, $r_{(63)}=0.53, p<0.01$, anxiety. Individuals who reported greater alexithymia also reported greater anxiety.

Two-way analyses of variance with country and gender as the independent variables were performed on 
the TAS and STAI scores. The Kyrgyz participants showed a significantly higher rate of alexithymia, $F_{(1,60)}=$ 23.62, $p<0.01$. No country differences were found for state or trait anxiety. No gender differences or interaction effects were found for either the TAS or STAI scores.

Multiple regression analysis was used to determine whether country, state or trait anxiety (STAI scores) was the better predictor of alexithymia (TAS scores). The linear regression analysis with alexithymia as the dependent variable and country (dummy coded 0 and 1 ) and state and trait anxiety entered as predictors was significant, $R^{2}=0.49, F_{(3,60)}=19.04, p<0.01$. Country was found to predict alexithymia (TAS scores), even when the STAI state and trait anxiety scores were controlled, $t=4.68, p<0.01$. Trait anxiety also predicted the alexithymia scores even when country and state anxiety was controlled, $t=3.19, p<0.01$; Beta $=0.38$.

\section{Discussion and Conclusions}

The results corroborate previous research on the connection between alexithymic characteristics and level of anxiety. However, an issue of culturally determined mechanisms for alexithymic characteristics is also found. The results provide evidence for the possibility of cultural roots of alexithymia. Alexithymia as a reduced capacity to identify and verbalize one's feelings is an expression of emotional openness/closedness. A greater occurrence of alexithymic characteristics in the Kyrgyz sample, and as a consequence less expressed emotional openness, might be seen as a product of social learning of the appropriate norms, beliefs and regulations of this culture.

The historical, social, and cultural framework of Kyrgyzstan, a Central Asia republic, is characterized by a merger of several traditional cultures: Nomadism, Soviet totalitarianism and Islam. A feature common in these three traditions is rigorous social control over emotional expression, which suppresses the ability to verbally describe one's own emotional experience. In this regard, however, alexithymia should not be discussed in terms of "inability" or "infringement", but rather a culturally-bounded phenomenon reflecting the population norm.

The American sample shows greater access to emotions. This result fits with the American cultural values of freedom of self-expression and lack of social pressure to modulate or regulate emotional behavior. The American sample is also more homogenous than Kyrgyz sample. The diversity of cultural traditions in Kyrgyzstan along with active westernization of the region may play a role in the development of a variety of emotional phenomena including the phenomenon of "cultural alexithymia".

\section{References}

Asmal, L., \& Stein, D. J. (2009). Anxiety and culture. In M. M. Antony, M. B. Stein, M. M. Antony, \& M. B. Stein (Eds.), Oxford handbook of anxiety and related disorders (pp. 657-664). New York, NY: Oxford University Press.

Bagby, R. M., Parker, J. D., \& Taylor, G. J. (1994). The twenty-item Toronto Alexithymia scale: Item selection and cross-validation of the factor structure. Journal of Psychosomatic Research, 38, 23-32.

Güleç, H. H., Köse, S. S., Güleç, M. Y., Çitak, S. S., Evren, C. C., Borckardt, J. J., \& Sayar, K. K. (2009). Reliability and factorial validity of the Turkish version of the 20-item Toronto Alexithymia Scale (TAS-20). Bulletin of Clinical Psychopharmacology, 19(3), 214-220.

Hanin, Y. L., \& Spielberger, C. D. (1983). The development and validation of the Russian form of the state-trait anxiety inventory. Series in Clinical \& Community Psychology: Stress and Anxiety, 215-226.

Karukivi, M., Hautala, L., Kaleva, O., Haapasalo-Pesu, K., Liuksila, P., Joukamaa, M., \& Saarijärvi, S. (2010). Alexithymia is associated with anxiety among adolescents. Journal of Affective Disorders, 125(1-3), 383-387. doi:10.1016/j.jad.2010.02.126

Le, H., Berenbaum, H., \& Raqhavan, C. (2002). Culture and alexithymia: Mean levels, correlates, and the role of parental socialization of emotions. Emotion, 2(4), 341-360. 
Nemiah, J. C., Freyberger, H., \& Sifneous, P. E. (1976). Alexithymia: A view of the psychosomatic process. In O. Hill (Ed.), Modern Trends in Psychosomatic Medicine (Vol. 3, pp. 430-439). London, Butterworths.

Parker, J. A., Taylor, G. J., \& Bagby, R. (1998). Alexithymia: Relationship with ego defense and coping styles. Comprehensive Psychiatry, 39(2), 91-98. doi:10.1016/S0010-440X(98)90084-0.

Salminen, J. K., Saarijärvi, S., Äärelä, E., Toikka, T., \& Kauhanen, J. (1999). Prevalence of alexithymia and its association with sociodemographic variables in the general population of Finland. Journal of Psychosomatic Research, 46(1), $75-82$. doi:10.1016/S0022-3999(98)00053-1

Spielberger, C. (1983). State-trait anxiety inventory for adults. Redwood City, C. A.: Mind Garden.

Taylor, G. J., Bagby, R., \& Parker, J. (1997). Disorders of affect regulation: Alexithymia in medical and psychiatric illness. New York: Cambridge University Press. doi:10.1017/CBO9780511526831

Taylor, G. J., Bagby, R., \& Parker, J. A. (2003). The 20-item Toronto Alexithymia Scale IV. Reliability and factorial validity in different languages and cultures. Journal of Psychosomatic Research, 55(3), 277-283. doi:10.1016/S0022-3999(02)00601-3

Taylor, G. J., Bagby, R., Ryan, D. P., \& Parker, J. D. (1988). Criterion validity of the Toronto Alexithymia Scale. Psychosomatic Medicine, 50(5), 500-509.

Vanheule, S., Desmet M., Megank, R., \& Bogarets, S. (2007). Alexithymia and interpersonal problems. Journal of Clinical Psychology, 63(1), 109-17. 